\title{
Prevalencia de Obesidad y Sobrepeso en Estudiantes de Primer Ingreso de la Carrera de Químico Biólogo Clínico de la Universidad de Sonora, Unidad Regional Sur
}

\section{"Prevalence of Obesity and overweight in first-year students of the Clinical Biologist Chemistry degree at the University of Sonora, South Regional Unit"}

Ruiz-López, Liliana 1*; Felipe-Ortega Fonseca, Ximena; Balderrama-Carmona, Ana Paola; Vázquez-Curiel, Rosa Amelia; Ruiz- Valdez, Alejandra Guadalupe; Reyes-Ruiz, Rosario Esperanza.

\section{Resumen}

La obesidad y sobrepeso se caracterizan por ser una enfermedad crónica con numerosas complicaciones de padecimientos con graves impactos en calidad de vida y de productividad. México en el 2018 presenta un $75.2 \%$ (39.1\% sobrepeso y $36.1 \%$ obesidad) en personas de 20 a más años; porcentaje que en 2012 fue de $71.3 \%$. En Sonora la prevalencia es de $22.2 \%$ ocupando el cuarto lugar en obesidad y sobrepeso para la población de 12 a 19 años. La mala alimentación aunado al sedentarismo potencializan la tendencia a padecer obesidad y sobre peso; que son factores de riesgo para muchas enfermedades como diabetes, hipertensión, cáncer. El ingreso de los adolescentes a la Universidad causa un desequilibrio en estilo de vida y hábitos alimenticios, lo que conlleva a problemas de salud derivado de una alimentación inadecuada; es por ello la importancia del monitoreo de Obesidad y sobrepeso en estudiantes universitarios; En esta investigación se determinó la prevalencia de obesidad y sobrepeso en los estudiantes de primer ingreso de la carrera de Químico Biólogo de la Universidad de Sonora Unidad Regional Sur, por medio un estudio descriptivo en donde participó una población de 124 estudiantes a los que se les determinó el IMC mediante evaluaciones antropométricas (EA) y análisis clínicos de colesterol triglicéridos y glucosa. Encontramos una prevalencia de obesidad y sobrepeso de $23.80 \%$; siendo ligeramente mayor a la publicada por ENSANUT 2018 dentro del mismo estado (22.2\%); así mismo se presentó prevalencia de obesidad (7.14\%) presentándose obesidad de tipo grado I (2.38 \%) y grado III (4.76\%); La relación estadística entre el IMC y la glucosa es de $99.9 \%(\mathrm{p} \leq 0.001)$, en cuanto a colesterol es del $99 \%(\mathrm{p} \leq 0.015)$ y triglicéridos tiene una significancia menor al 95\% ( $\mathrm{p} \leq 0.055)$. Por lo que podemos asociar tanto glucosa como colesterol con un problema de sobrepeso.

Palabras clave: Obesidad, Sobrepeso, Universitarios.

Dpto. de Cs. Químico-Biológicas y Agropecuarias. Lázaro Cárdenas \#100 Col. Francisco. Villa. CP. 85880. Navojoa, Sonora. Teléfono: 01(642) 4259969.

*Autor de correspondencia: M.C. Liliana Ruiz López; correo electrónico: liliana.ruizlopez@unison.mx; lruiz@navojoa.uson.mx 


\title{
http://revistainvestigacionacademicasinfrontera.com
}

\begin{abstract}
Obesity and overweight are characterized as a chronic disease with numerous complications of conditions with serious impacts on quality of life and productivity. Mexico in 2018 is $75.2 \%$ (39.1\% overweight and 36.1\% obese) in people 20 years of age and older; percentage that in 2012 was $71.3 \%$. In Sonora, the prevalence is $22.2 \%$ occupying the fourth place in obesity and overweight for the population of 12 to 19 years. Poor diet coupled with sedentary lifestyle potentiate the tendency to suffer from obesity and overweight; which are risk factors for many diseases such as diabetes, hypertension, cancer. The entrance of adolescents to the University causes an imbalance in lifestyle and eating habits, which leads to health problems derived from improper feeding; that is why the importance of monitoring obesity and overweight in university students; In this investigation, the prevalence of obesity and overweight was determined in the first-year students of the Biologist Chemistry degree at the University of Sonora South Regional Unit, through a descriptive study in which a population of 124 students participated. Determined BMI by anthropometric evaluations (EA) and clinical analyzes of triglyceride cholesterol and glucose. We found a prevalence of obesity and overweight of $23.80 \%$; being slightly higher than the one published by ENSANUT 2018 within the same state (22.2\%); Likewise, there was a prevalence of obesity $(7.14 \%)$, with obesity of type grade I $(2.38 \%)$ and grade III (4.76\%); The statistical relationship between BMI and glucose is $99.9 \%(\mathrm{p} \leq 0.001)$, in terms of cholesterol it is $99 \%(\mathrm{p} \leq 0.015)$ and triglycerides have a significance of less than $95 \%(\mathrm{p} \leq 0.055)$. Therefore, we can associate both glucose and cholesterol with an overweight problem.
\end{abstract}

Keywords: Obesity, Overweight, University students

\section{Introducción}

La obesidad es una enfermedad de etiología multifactorial de curso crónico en la cual se involucran aspectos genéticos, ambientales y de estilo de vida. Se caracteriza por un balance positivo de energía, que ocurre cuando la ingestión de calorías excede al gasto energético, ocasionando un aumento en los depósitos de grasa corporal y ganancia de peso ${ }^{(9)}$. La obesidad es un problema de salud pública, que requiere prevención, detección temprana, tratamiento integral, control y disminución de casos. De acuerdo con la Organización Mundial de la Salud (OMS), el sobrepeso y la obesidad son enfermedades crónicas. Ambas, se acompañan de alteraciones metabólicas y 


\section{http://revistainvestigacionacademicasinfrontera.com}

nutricionales que incrementan el riesgo para desarrollar comorbilidades tales como: hipertensión arterial, diabetes mellitus tipo 2, enfermedades cardiovasculares y cerebrovasculares, así como algunas neoplasias en mama, endometrio, colon y próstata y otros padecimientos discapacidades que afectan la calidad de vida del que lo padece ${ }^{(1)}$. Por esta razón, es un problema prioritario que requiere de atención y acciones intersectoriales inmediatas para prevención en la población ${ }^{(9)}$. El Acuerdo Nacional para la Salud Alimentaria (ANSA) de México, firmado en 2010 ha sido el primer esfuerzo que contó con una política explicita para la prevención de la obesidad y sus enfermedades crónicas asociadas ${ }^{(3)}$.

Es importante considerar que la obesidad no es una enfermedad exclusiva de los adultos, ya que tanto en la población infantil (5 y 11 años) como en la adolescente (12 a 19 años) se ha presentado un alto crecimiento durante los últimos años, por lo que en el año 2010 nos llevó al segundo lugar a nivel mundial en obesidad en adultos y el primero en infantil ${ }^{(2)}$.

La globalización y la comercialización de la industria alimentaria con políticas de alimentos y dieta de acuerdo al el poder adquisitivo de la sociedad ${ }^{(8)}$; ha impulsado al cambio de alimentación sana por productos procesados, que no siempre cumplen con los requerimientos dietéticos de la población, lo cual lleva a un cambio en los patrones alimenticios de las familias aunado a los estilos de vida propios de la modernidad, así como las largas jornadas laborales que han llevado a una disminución de la actividad física y aumento del sedentarismo en la sociedad ${ }^{(2)}$; han sido factores importantes y propicios para el aumento en el número de pacientes que presentan sobrepeso y obesidad.

La Obesidad es una enfermedad muy compleja y poco entendida. La Organización Mundial de la Salud (OMS), para describir dicha condición, utiliza las dimensiones del cuerpo, recurriendo al índice de masa corporal (IMC) como el mejor indicador de la grasa corporal. Por lo que, individuos con un IMC entre 26 y 29.9 se consideran con Sobrepeso, aquellos que exceden de un IMC de 30 se les denomina sujetos con Obesidad, los que rebasan los 40 se padecen de Obesidad mórbida y los que sobrepasan los 50 se les considera que padecen Obesidad extrema ${ }^{(4,6,10)}$. 


\section{http://revistainvestigacionacademicasinfrontera.com}

En México en el 2018 el 17.2\% de la población de 0 a 4 años presentó sobrepeso, en edades de 5 a 11 años un $35.8 \%$ en sobrepeso y $31.8 \%$ obesidad, por otro lado edades de 12 a 19 años presentan $45.7 \%$ de sobrepeso y $28.6 \%$ de obesidad; en adultos con más de 20 años se presentó $75.2 \%$ (39.1\% sobrepeso y 36.1\% obesidad). En Sonora los resultados de la ENSANUT 2018 informan una prevalencia del $22.2 \%$ de Sobrepeso y Obesidad en la población sonorense mayor a los 20 años ${ }^{(5)}$.

El ingreso de los adolescentes a la universidad causa un desequilibrio en estilo de vida y hábitos alimenticios, caracterizados por inadecuada alimentación fuera de casa como la ingesta de alimentos chatarra o mal balanceados ${ }^{(7)}$. Otra condicionante de la mala nutrición estudiantil de debe a la falta de tiempo para preparar sus alimentos debido a horarios inapropiados por la carga académica; y a que la mayor parte de los estudiantes en la Unidad Regional Sur pertenecen a comunidades rurales e indígenas y con escasos recursos económicos; por lo que en algunos casos su alimentación es deficiente nutrimentalmente, lo cual repercute directamente en su salud y desempeño académico. En nuestro país existen pocos estudios en estudiantes de nuevo ingreso a la Universidad; por lo que es importante determinar el estado de salud de nuestros estudiantes de primer ingreso y detectar a los alumnos de mayor vulnerabilidad a padecer sobrepeso y obesidad.

Objetivo: Determinar la prevalencia de sobrepeso y obesidad en estudiantes de primer ingreso de la carrera de Químico Biólogo Clínico de la Universidad de Sonora Unidad Regional Sur.

\section{Metodología}

Durante el segundo periodo del 2018 de un total de 124 estudiantes de nuevo ingreso de la carrera de Químico Biólogo Clínico de la Universidad de Sonora Unidad Regional Sur, se tomó una muestra representativa con un nivel de confianza del $80 \%$, frecuencia del $25 \%$ y margen de error aceptable del 7\% (EPI INFOTM, 2017). A los participantes se les explicó verbalmente que son parte de un proyecto de investigación, en qué consiste éste, cuál es el beneficio y cuál es el sentido de reportar los resultados, también se les comentó que su participación sería voluntaria y gratuita, se aplicó encuesta que incluyó características generales. También se explicó que su identidad, datos 
Núm. 28

ISSN: 2007-8870

\section{http://revistainvestigacionacademicasinfrontera.com}

y resultados de la evaluación antropométrica y clínica se mantendrán bajo confidencialidad teniendo acceso solo el participante y el personal de salud.

\section{Evaluación antropométrica}

Todas las mediciones se llevaron a cabo en el consultorio de nutrición por personal entrenado. El peso fue medido usando una balanza electrónica (Tanita, Body Composition Analizer BF 350) con bioimpedancia eléctrica de acuerdo a procedimientos estandarizados. Para la altura se usó un estadiómetro con una longitud máxima de 2.07 m (Invicta Plastics, Ltd). El índice de masa corporal (IMC) de los estudiantes fue calculado del resultado de dividir el peso (en $\mathrm{kg}$ ) entre la talla (expresada en metros) al cuadrado ( $\mathrm{kg} / \mathrm{m} 2)$. A los participantes se les entregaron los resultados directamente del responsable de nutrición, para lo cual se les brindó explicación y apoyo en el caso que se requiriera.

\section{Aplicación de encuesta}

A los participantes se les aplicó encuesta que incluyó características generales (Edad, género, peso, estatura, enfermedades o padecimientos específicos). Se realizó la investigación respetando lo establecido por la Ley General de Salud, en materia de investigación en seres humanos. La participación fue de manera voluntaria a las pruebas antes mencionadas, ellos firmaron una carta de consentimiento informado y se les explicó la naturaleza, el propósito y beneficios de dicho estudio, también se les explicó sobre todo la confidencialidad de los datos o información obtenida.

\section{Toma de muestras biológicas}

Se realizó la toma de muestra utilizando el sistema Vacutainer mediante punción venosa a los alumnos participantes, utilizando tubos con anticoagulante EDTA marca Vacutainer, para el 
Núm. 28

ISSN: 2007-8870

\section{http://revistainvestigacionacademicasinfrontera.com}

análisis clínico. Las muestras fueron tomadas y analizadas dentro de las instalaciones del laboratorio clínico de Servicio a la Comunidad de la Universidad de Sonora URS.

\section{Análisis Clínicos}

Todas las mediciones y análisis clínicos se llevaron a cabo y de forma privada en el Laboratorio de Servicios a la Comunidad de la Universidad de Sonora. Los datos que se obtuvieron se incluyen en el proyecto únicamente si existe un consentimiento firmado de cada uno de los participantes. Se realizaron análisis clínicos consistentes en Perfil lipídico (colesterol, triglicéridos) y glucosa, para los que se utilizó el equipo BS-200 Analizador Químico, Marca Mindray, empleando calibradores de la marca Elical y sueros control bajo normal y alto marca Elitrol (Francia) tales como: Elitrol I, Elitrol II, Cholesterol Sl, Triglycerides Mono S1 New, Glucose Pap S, para detectar los metabolitos de colesterol, triglicéridos y glucosa. Para realizar la separación de sueros de las muestras sanguíneas se utilizó una Centrifuga Modelo 80-2s, y a una velocidad de 3000 rpm por 5 minutos. El desecho de las muestras biológicas y material empleado para la toma de muestra fue realizado siguiendo los lineamientos de la NOM-087-SEMARNAT-SSA1-2002. Los resultados obtenidos en el procesamiento de las muestras sanguíneas y el diagnóstico fueron entregados a los participantes directamente del personal de salud, también se invitó a los participantes que tuvieran dudas o que necesiten tratamiento a informarse en el consultorio médico de la Universidad de Sonora, Unidad Regional Sur y sean canalizados a su centro de salud que les corresponda. La emisión del diagnóstico y el establecimiento del tratamiento fueron responsabilidad del médico de la institución de salud que atendió a cada participante.

\section{Análisis estadístico}

El tamaño de la población del estudio y los análisis para de regresión lineal múltiple fueron desarrollados el software estadístico IBM SPSS. 


\section{http://revistainvestigacionacademicasinfrontera.com}

\section{Resultados}

En la figura 1 se muestra que la población evaluada está constituida por mujeres en un $71.43 \%$ y varones con un $28.57 \%$.

\section{POBLACIÓN DE ESTUDIO POR SEXO}

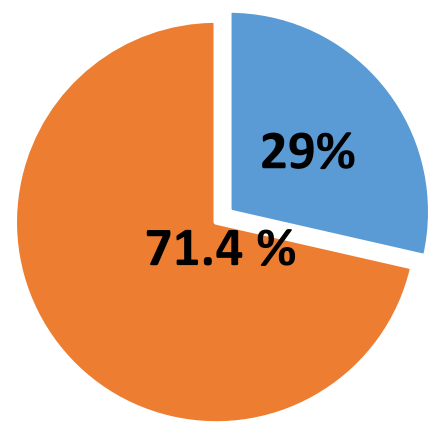

- Masculino Femenino

Figura 1. Porcentaje de Población de Estudio por Sexo

\section{Evaluación antropométrica}

En la Tabla 1, se muestran las características antropométricas de la población de estudio. La media del IMC para los alumnos de 18 años fue de 23.40; en la edad de 19 años se presentó una media de 35.93; para la edad de 20 años fue de 23.46, para 21 años fue de 21.27 y se observó un IMC muy elevado de 41.03 en la edad de 22 años. 
Núm. 28

http://revistainvestigacionacademicasinfrontera.com

Tabla 1. Índice de Masa Corporal (IMC) por edades

\begin{tabular}{cccccccccc}
\hline Años & $\begin{array}{c}\text { Peso } \\
(\mathbf{K g})\end{array}$ & Mínimo & Máximo & $\begin{array}{c}\text { Talla } \\
(\mathbf{c m})\end{array}$ & Mínimo & Máximo & IMC & Mínimo & Máximo \\
& Media & & & & & & & \\
& & & & & & & & & \\
& & & & & & & & & \\
18 & 60.66 & 50 & 80.2 & 161.5 & 150 & 187 & 23.40 & 22.22 & 22.93 \\
19 & 66.46 & 48 & 151.9 & 135.9 & 155 & 184 & 35.93 & 19.98 & 44.87 \\
20 & 67.00 & 65.7 & 68.3 & 169.5 & 161 & 178 & 23.46 & 25.35 & 21.56 \\
21 & 59.33 & 48 & 70 & 166.7 & 159 & 173 & 21.27 & 17.21 & 23.39 \\
22 & 130 & 130 & 130 & 178 & 178 & 178 & 41.03 & 41.03 & 41.03 \\
\hline
\end{tabular}

\section{Sobrepeso y tipos de obesidad}

De acuerdo al IMC determinado y en base a la clasificación de la OMS, se observa que en este estudio Se tiene una prevalencia de sobrepeso y obesidad de 23.80\%; así mismo se encontró que un $73.84 \%$ de los participantes presentan un peso adecuado; mientras que el $2.38 \%$ de los estudiantes presentaron bajo peso y un $16.66 \%$ sobrepeso. Dentro de los estudiantes que presentaron obesidad se encuentra un $2.38 \%$ con obesidad grado I y un $4.76 \%$ con obesidad grado III (Tabla 2). 
Núm. 28

ISSN: 2007-8870

http://revistainvestigacionacademicasinfrontera.com

Tabla 2. Porcentaje en Peso según la clasificación del IMC de la OMS.

\begin{tabular}{lll}
\hline Clasificación & $\%$ & Valores de Referencia (OMS) \\
Bajo Peso & 2.38 & 18.5 \\
Peso Normal & 73.84 & $18.5-24.9$ \\
Sobrepeso & 16.66 & $25-29.9$ \\
Obesidad Grado I & 2.38 & $30-34.9$ \\
Obesidad Grado II & 0 & $35-39.9$ \\
Obesidad Grado III & 4.76 & $>40$ \\
\hline
\end{tabular}

Padecimientos en estudiantes de primer ingreso de la carrera de Químico Biólogo Clínico de la Universidad de Sonora, Unidad Regional Sur.

Los resultados obtenidos hacen referencia a la aplicación de una encuesta estructurada en las instalaciones de la Universidad de Sonora Unidad Regional Sur. Durante las encuestas aplicadas obtuvimos la siguiente información; el $23.80 \%$ no presentó padecimientos; el $9.52 \%$ han sentido fatiga recientemente durante sus actividades cotidianas, $4.76 \%$ presentan problemas de diabetes, el $35.71 \%$ de los alumnos tienden a sentirse con dolores de cabeza o migraña, el $14.29 \%$ de los estudiantes padecen de enfermedades gastrointestinales y únicamente el $2.38 \%$ padece de problemas cardiovasculares y niveles altos de colesterol (Tabla 3). 


\section{http://revistainvestigacionacademicasinfrontera.com}

Tabla 3. Padecimientos en alumnos de primer ingreso de la carrera de Químico Biólogo Clínico.

\section{Padecimientos}

Problemas cardiovasculares

Diabetes

Niveles altos de colesterol

Fatiga

Dolores de cabeza, migraña

Desórdenes gastrointestinales

Ninguna

Otras

\section{$\%$}

2.38

4.76

2.38

9.52

35.71

9.52

\section{Análisis estadístico}

La significancia estadística entre el IMC y la glucosa es de 99.9\% ( $\mathrm{p} \leq 0.001)$, en cuanto a colesterol es del $99 \%(\mathrm{p} \leq 0.015)$ y triglicéridos tiene una significancia menor al $95 \%(\mathrm{p} \leq 0.055)$. Por lo que podemos asociar tanto glucosa como colesterol con un problema de sobrepeso, esta premisa se puede reforzar con el estudio realizado por Onyesom y cols. ${ }^{11}$ donde señalan que índices de masa corporal altos pueden relacionarse con los niveles de glucosa sanguínea en mujeres, también se ha correlacionado positivamente el IMC con niveles de colesterol total ${ }^{12}$.

\section{Conclusiones}

Una vez concluido el presente proyecto de investigación se encontró una prevalencia de obesidad y sobrepeso de 23.80\%; siendo ligeramente mayor a la publicada por ENSANUT 2018 dentro del mismo estado (22.2\%); así mismo se presentó prevalencia de obesidad (7.14\%) presentándose obesidad de tipo grado I (2.38 \%) y grado III (4.76\%); La relación estadística entre el IMC y la glucosa es de 99.9\% ( $\mathrm{p} \leq 0.001)$, en cuanto a colesterol es del 99\% $(\mathrm{p} \leq 0.015)$ y triglicéridos tiene una significancia menor al 95\% ( $\leq 0.055)$. Por lo que podemos asociar tanto glucosa como 


\section{http://revistainvestigacionacademicasinfrontera.com}

colesterol con un problema de sobrepeso. Los padecimientos más comunes producto de la encuesta en la comunidad estudiantil fueron dolores de cabeza (35.71\%), desórdenes gastrointestinales (14.29\%), fatiga (9.52\%), diabetes (4.76\%), niveles altos de colesterol (2.38\%) y problemas cardiovasculares $(2.38 \%)$ los cuales pueden considerarse como indicativos de la mala alimentación de los estudiantes. Por lo que se recomienda implementar pláticas, programas de nutrición y activación física; que apoye en mejorar las condiciones de salud en los estudiantes de la Universidad de Sonora Unidad Regional Sur.

\section{Agradecimientos}

Se agradece a los QBC Isaac Mendoza López, María Esther Félix Valenzuela, Esteban Padilla Valdez por su valiosa participación como prestadores de servicio social en este proyecto y a la M.C. Gabriela de los Ángeles Díaz Reyes por su apoyo brindado en el Laboratorio de Servicio a la Comunidad de la Universidad de Sonora Unidad Regional Sur.

\section{Bibliografía}

1.-Acebo Martínez, M. L. (2017). Obesidad y salud, ¿En realidad existe el paciente metabólicamente obeso? Revista Salud Pública y Nutrición, 16(2), 44-55. Recuperado de http://respyn.uanl.mx/index.php/respyn/article/view/33/170

2.- Barroso C, C. (2012). La obesidad, un problema de salud pública. Espacios Públicos, vol. 15, núm. 33, enero-abril, 2012, pp. 200-215. Universidad Autónoma del Estado de México Toluca, México

Disponible en: http://www.redalyc.org/articulo.oa?id=67622579011 


\section{http://revistainvestigacionacademicasinfrontera.com}

3.- Barquera S, Campos-Nonato I, Hernandez-Barrera L, Pedroza A, Rivera-Dommarco JA. (2013). Prevalencia de obesidad en adultos mexicanos, Ensanut 2012. Salud Pública Mex. 2013; 55(Suppl 2):S151-60. https://doi. org/10.21149/spm.V55s2.5111.

4.- Definition and diagnosis of diabetes mellitus and intermediate hyperglycemia: report of a WHO/IDF consultation. Geneva, Switzerland: WHO/IDF 2006.

5.- Encuesta Nacional de Salud y Nutrición 2018. Presentación de resultados. Secretaría de Salud, Instituto Nacional de Salud Pública (INSP), Instituto Nacional de Estadística y Geografía (INEGI). Disponible en: http://www.inegi.org.mx > $2018>$ doc > ensanut_2018_presentacion_resultados

6.- Pan American Health Organization (2000). Obesity and poverty: a new public health challenge. Washington DC: PAHO, 2000 [citado noviembre 1, 2019]. Disponible en: http://new.paho.org/hq/dmdocuments/2010/9275115761.pdf

7.- Sáenz Duran S y Cols. (2011). Hábitos y trastornos alimenticios asociados a factores sociodemográficos, físicos y conductuales en Universitarios de Cartagena, Colombia. Revista clínica de Medicina de familia; 4(3):193-204. Versión Online ISSN 2386-8201 Versión impresa ISSN 1699-695x

8.- Shamah-Levy T, Campos-Nonato I, Cuevas-Nasu L, Hernández-Barrera L, Morales-Ruán MC, Rivera-Dommarco J, Barquera S (2019). Sobrepeso y obesidad en población mexicana en condición de vulnerabilidad. Resultados de la Ensanut 100k. Salud Pública Mex. 2019; 61:852865. https://doi.org/10.21149/10585

9.- Torres-Zapata AE., Zapata-Gerónimo D., Rivera-Domínguez J., Acuña-Lara JP. (2018) El obeso de peso normal, 17(2), 25-31. 
Núm. 28

ISSN: 2007-8870

\section{http://revistainvestigacionacademicasinfrontera.com}

DOI: https://doi.org/10.29105/respyn17.2-4

10.- WHO. Obesity: Preventing and managing the global epidemic. Report of a WHO consultation Ginebra: WHO.2000 (Technical Report Series 894); 203.

ISBN 9241208945

ISSN 0512-3054

11.- Onyesom I, Oweh O, Etumah O S and Ifie E J. (2013) Correlation between body mass index and blood glucose levels among some Nigerian undergraduates. HOAJ Biology. DOI: http://dx.doi.org/10.7243/2050-0874-2-4

12.- Avnish D, Makwana J, Thomas E. (2018) Waist Height Ratio (WHTR), Body Mass Index (BMI) and Serum Cholesterol. International Journal of Basic and Applied Physiology, 7(1): 105110. 
"El saber de mis hijos
hará mi grandeza"

Año 11

Núm. 28

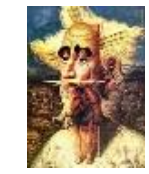

Revista de Investigación Académica sin Frontera ISSN: 2007-8870

\section{http://revistainvestigacionacademicasinfrontera.com}

Apéndice

Evaluación Antropométrica
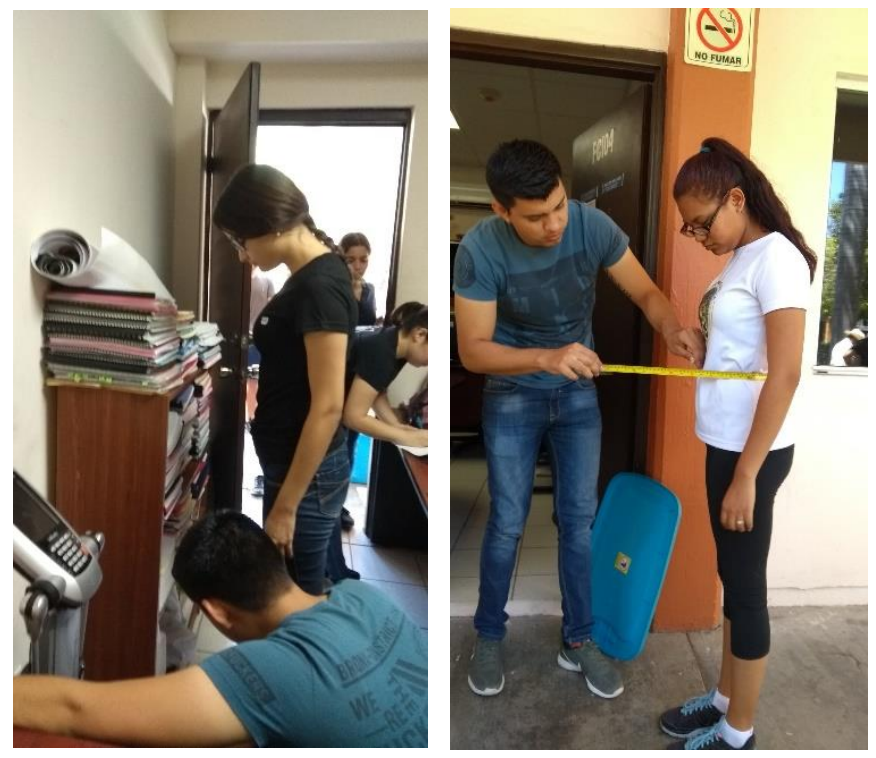

Firma de consentimiento informado y toma de muestra
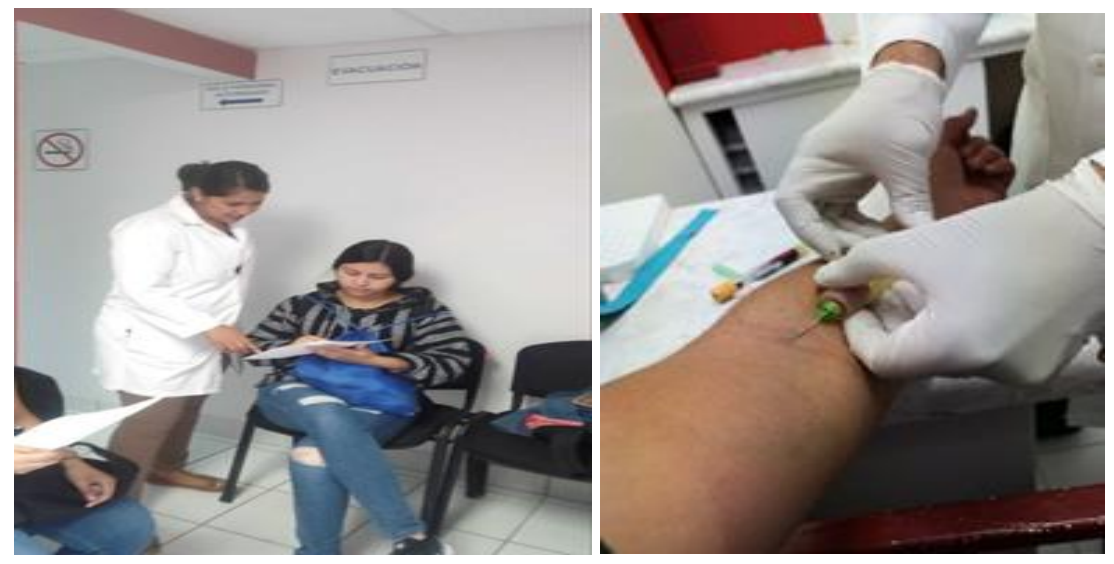